\title{
Transplant trials with Tregs: perils and promises
}

\author{
Qizhi Tang ${ }^{1,2}$ and Flavio Vincenti $i^{1,3}$ \\ 'Department of Surgery, ${ }^{1}$ Diabetes Center, and ${ }^{3}$ Department of Medicine, UCSF, San Francisco, California, USA.
}

\begin{abstract}
Modern immunosuppression regimens effectively control acute rejection and decrease graft loss in the first year after transplantation; however, these regimens do not have a durable effect on long-term graft survival owing to a combination of drug toxicities and the emergence of chronic alloimmune responses. Eliminating drugs and their toxicities while maintaining graft acceptance has been the primary aim of cellular therapies. Tregs suppress both autoimmune and alloimmune responses and are particularly effective in protecting allografts in experimental transplant models. Further, Treg-based therapies are selective, do not require harsh conditioning, and do not have a risk of graft-versus-host disease. Trial designs should consider the distinct immunological features of each transplanted organ, Treg preparations, dose, and frequency, and the ability to detect and quantify Treg effects in a given transplant environment. In this Review, we detail the ongoing clinical trials of Treg therapy in liver and kidney transplantation. Integration of Treg biology gleaned from preclinical models and experiences in human organ transplantation should allow for optimization of trial design that will determine the potential efficacy of a given therapy and provide guidelines for further therapeutic development.
\end{abstract}

\section{The quest for transplantation tolerance}

The quest for successful, drug-free organ transplantation, which can be achieved routinely in identical twins who are kidney donor/ recipient pairs, remains challenging for all other patients. The few published successes have been achieved in recipients who have developed tolerance after receiving stem cells and/or bone marrow cells from the same kidney donor (1), and spontaneous tolerance has been achieved in a minority of liver transplant recipients (2). Achieving tolerance by inducing chimerism with stem cells poses major risks from the conditioning regimen, the instability of the drug-free tolerant state, and the possibility of developing graftversus-host disease (GVHD) (3). A more nuanced approach to tolerize recipients to allografts is to expand the pool of Tregs in order to harness their ability to control alloimmune responses $(4,5)$.

Decreased dependence on calcineurin inhibitor-based (CNIbased) regimens is a desirable goal in kidney transplantation. CNIs produce effective immunosuppression early after transplantation, but have adverse long-term effects for both the patient and the renal allograft (6). CNIs impede the emergence of tolerogenic immune responses, propagating the dependence on maintenance drugs for immunosuppression $(7,8)$. Unlike currently used experimental stem cell regimens, Treg therapies do not require drastic conditioning regimens and do not pose a risk of $\operatorname{GVHD}(4,9)$; however, the promising results obtained with Treg infusions in experimental transplantation and autoimmunity models have yet to be fully tested in humans. In this Review, we summarize the rationale for using Treg therapy in transplantation and describe currently

Conflict of interest: Q. Tang is an inventor on a patent (US patent 7,722,862,B2) and a provisional patent (US provisional patent 20150110761 A1) on Treg cell therapy. Q. Tang received a research grant to support Treg cell therapy development from Caladrius Biosciences Inc. and Juno Therapeutics. F. Vincenti has received research grants from Bristol-

Myers Squibb, Novartis, Astellas, Alexion, and Genentech.

Reference information: J Clin Invest. 2017;127(7):2505-2512.

https://doi.org/10.1172/JCI90598. used protocols and future strategies to enhance the potential of Treg-based therapies.

\section{Tregs in transplantation in preclinical models}

Tregs control the activities of a variety of immune cells in vivo (10, 11). Under steady-state conditions, Tregs serve as an "IL-2 sink" by virtue of their constitutive expression of CD25, which enables high-affinity binding of IL-2. Low amounts of IL-2 produced during low-grade immune activation are preferentially consumed by Tregs, boosting Treg homeostasis and preventing overt immune activation (12). Additionally, Tregs constitutively express cytotoxic T lymphocyte antigen-4 (CTLA-4), which can snatch the costimulatory ligands CD80 and CD86 from the surface of antigen presenting cells (APCs), thereby raising the threshold for $\mathrm{T}$ cell activation (13). Infections and inflammation during an active immune response increase the stimulatory capacity of APCs, overcoming Treg control and allowing an immune response to ensue. $\mathrm{T}$ cell receptor stimulation of Tregs by cognate antigens during an active immune response augments Treg suppression by increasing their steady-state functions and inducing an expanded array of suppressive mechanisms, including production of the immunosuppressive molecules IL-10, IL-35, TGF- $\beta$, and cAMP; expression of ectoenzymes CD39 and CD73 to degrade extracellular ATP; and expression of granzymes and perforin for direct killing of APCs (14). Thus, Tregs prevent expansion of conventional T cells (Tconvs) and prevent their acquisition of effector function.

Once activated, Tregs traffic to sites of inflammation, where they suppress immune cell effector functions and limit collateral tissue destruction (15). Depending on the context of their activation, Tregs develop the ability to suppress certain effector functions. For example, in the context of a Th1-mediated immune response, Tregs acquire a Th1-like phenotype by expression of the archetypal Th1 molecules IFN- $\gamma$ and CXCR3, allowing them to suppress the function of Th1 and CD8 ${ }^{+}$effector T cells (Teffs). Similarly, Tregs can specialize to suppress Th2, Th17, and T follicular helper cells. 
A

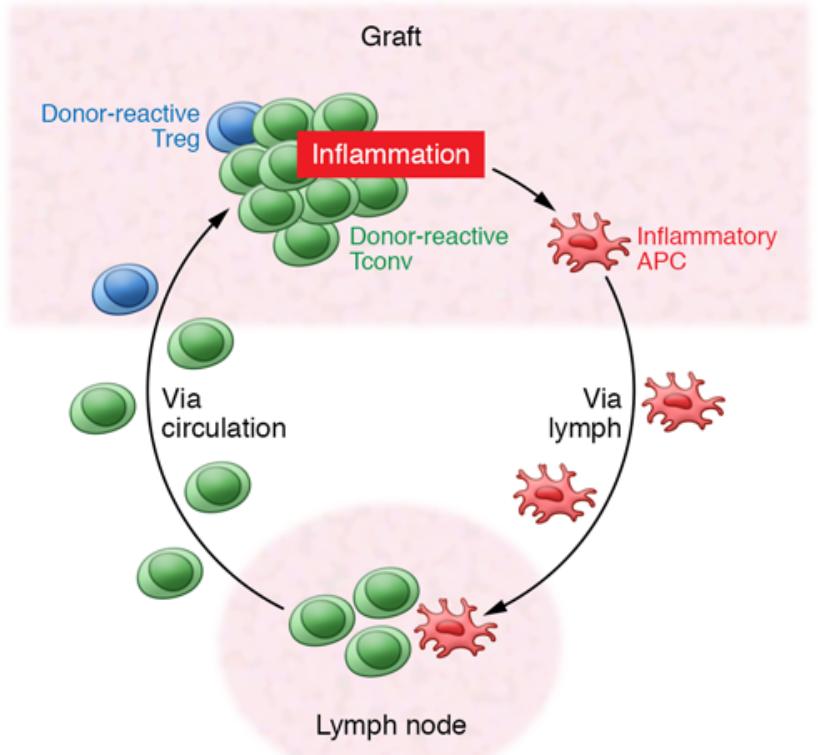

B

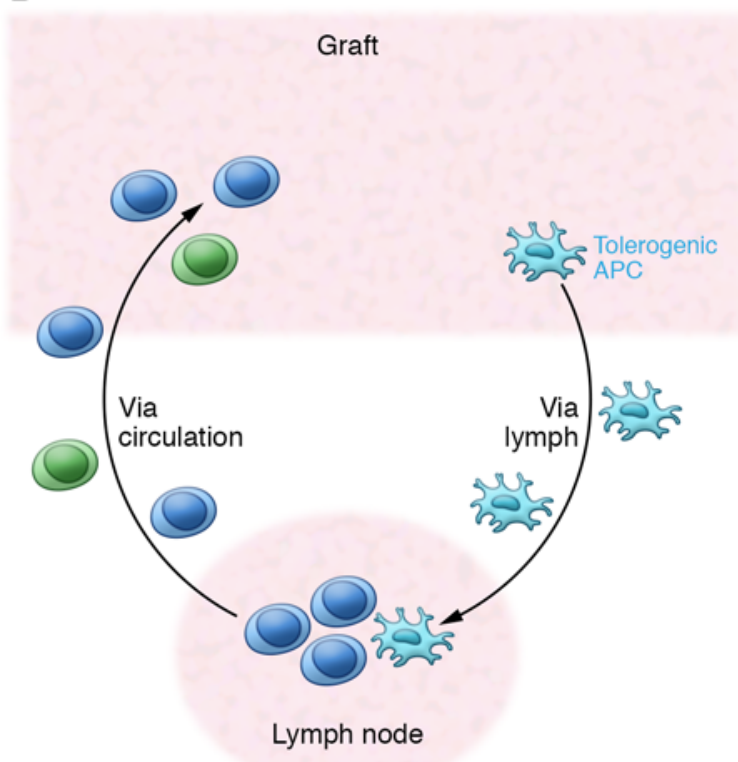

Figure 1. Anatomy of graft rejection and tolerance. (A) Vicious cycle of graft rejection. Graft-specific Tconvs enter the graft, leading to inflammation and tissue damage. Inflammatory antigen-presenting cells (APCs) enter the draining lymph node to activate more graft-specific cells. Graft-specific Tregs are too few to effectively control this process. (B) Increasing Tregs combined with reduction of graft-specific T cells allow Tregs to dominate in the graft and prevent recruitment and activation of effector T cells. Tolerogenic APCs enter the draining lymph node to expand graft-specific Tregs and prevent expansion of graft-specific T cells to maintain tolerance.

Many of these general principles of Treg function in controlling immune responses apply to Treg function in the context of alloimmune responses. T cells can recognize allogeneic MHC directly on donor cells or indirectly as antigenic fragments presented by host APCs (16). Tconvs capable of direct alloantigen recognition are present at a very high frequency so that they can respond to the transplant without first clonally expanding in lymph nodes. Direct alloantigen-reactive Tregs are also present at high frequency (17). In murine models, Tregs control transplant rejection by first migrating to the organ to limit graft damage and then retreating to draining lymph nodes to maintain tolerance (Figure 1 and ref. 18). Notably, Treg specificity for the induction and maintenance of tolerance may be distinct (19). Tregs with direct alloantigen specificity are important for the induction of tolerance, whereas Tregs with indirect alloantigen specificity are important for the maintenance of tolerance. Control of rejection and suppression of inflammation during the inductive phase not only serves to protect against graft damage, but also allows indirect Tregs to expand and establish long-term tolerance (Figure 1).

Tregs function in two distinct phases of immune responses. During homeostasis, Tregs prevent low-grade immune stimulation from developing into an overt immune response. During the resolution phase of an immune response, Tregs terminate immune effector functions. At the peak of an immune response, a high antigen load, vigorous costimulation, and high concentrations of cytokines such as IL- 1 and IL- 6 override Treg suppression so that effective immune functions can be carried out. The homeostatic function of Tregs is insufficient to prevent rejection from occurring due to the potency of alloimmune responses. During an active alloimmune response, Tregs of both direct and indirect speci- ficities expand and infiltrate the organs, but are usually unable to fully control the responses until the organs have suffered substantial damage $(20,21)$. Prevention of rejection and establishment of tolerance by Tregs require attenuation of Teff responses and inflammation control. For example, in a mouse islet transplantation model, Fan and colleagues monitored the dynamics of Treg and Teff responses in vivo and found that Teffs arrive at the graft site first and expand in number before the arrival of Tregs (22); thus, the grafts are dominated by Teffs and are quickly rejected. Treatment of the recipients with a tolerogenic regimen consisting of short-term CD4OL blockade and sirolimus greatly reduced Teff graft infiltration without affecting Treg infiltration, allowing Tregs to dominate in the graft site and preventing recruitment and accumulation of Teffs, thereby establishing tolerance. Raimondi and colleagues combined short-term sirolimus treatment with delayed Treg administration to effectively induce tolerance in a murine heart transplantation model (23). By transfusing recipients with donor alloantigen-reactive Tregs and treating with cyclophosphamide, Lee and colleagues preemptively reduced the donorreactive $\mathrm{T}$ cell frequency by $70 \%-80 \%$, inducing tolerance without the need for other immunosuppression (24). These preclinical results demonstrate that selective augmentation of Tregs can be an effective strategy for promoting transplantation tolerance.

Several lessons from mouse models are informative for the design of human Treg therapy trials. First, donor alloantigenspecific Tregs are more effective than polyclonal Tregs. When these two types of Tregs were compared head to head, alloantigenspecific Tregs were five to ten times more effective than polyclonal Tregs, with efficacy corresponding to the frequency of direct alloantigen-specific Tregs in the polyclonal Treg pool (24). This find- 


\section{Table 1. Reported or registered clinical trials of Treg cell therapy}

\begin{tabular}{|c|c|c|c|}
\hline Targeted enrollment & Type of Tregs & Type of trials & Trial ID \\
\hline \multicolumn{4}{|l|}{ Liver trials } \\
\hline 10 & $\begin{array}{l}\text { Autologous Tregs stimulated with irradiated donor } \\
\text { PBMCs with costimulation blockade }\end{array}$ & Phase 2 & Ref. 25, Japan \\
\hline 24 & Autologous donor antigen-expanded Tregs & Phase 1 & deLTa-UCSF, NCT02188719 \\
\hline 10 & Autologous polyclonally expanded Tregs & Phase $1 \& 2$ & Nanjing, China, NCT01624077 \\
\hline 18 & Autologous donor antigen-expanded Tregs & Phase $1 \& 2$ & ARTEMIS-UCSF, NCT02474199 \\
\hline 26 & Autologous polyclonally expanded Tregs & Phase $1 \& 2$ & ThRIL-KCL, NCT02166177 \\
\hline \multicolumn{4}{|l|}{ Kidney trials } \\
\hline 3 & Autologous polyclonally expanded Tregs & Phase 1 & TASKp-UCSF, NCT02088931 \\
\hline 45 & $\begin{array}{c}\text { Autologous polyclonally or donor antigen- } \\
\text { expanded Tregs }\end{array}$ & Phase $1 \& 2$ & TASK-UCSF, NCT02711826 \\
\hline 10 & Autologous polyclonally expanded Tregs & Phase 1 & TRACT-Northwestern, NCT02145325 \\
\hline 8 & $\begin{array}{l}\text { Autologous Tregs stimulated with irradiated donor } \\
\text { PBMCs with costimulation blockade }\end{array}$ & Phase 1 & One Study-MGH, NCT02091232 \\
\hline 12 & Autologous polyclonally expanded Tregs & Phase 1 & One Study-UK, NCT02129881 \\
\hline 9 & Autologous polyclonally expanded Tregs & Phase $1 \& 2$ & One Study-Charité, NCT02371434 \\
\hline 8 & Autologous donor antigen-expanded Tregs & Phase 1 & One Study-UCSF, NCT02244801 \\
\hline
\end{tabular}

ing suggests that donor alloantigen-specific Tregs are ideal for their greater potency and lower nonspecific immunosuppression, whereas polyclonal Tregs that are easier to manufacture may be effective in large doses. Second, Tregs are capable of dominant suppression, meaning that Tregs of one antigen specificity are able to suppress Teffs of various distinct specificities. Thus, therapeutic Tregs do not have to recognize all possible major and minor histocompatibility antigens to protect allografts. Third, Treg-induced tolerance can be maintained long-term through a process of "infectious tolerance," in which tolerance begets tolerance. Tregs specific to alloantigen A can induce tolerance to a graft that expresses both alloantigens A and B. Over time, alloantigen B-specific Tregs expand and can maintain tolerance in the absence of the original alloantigen A-specific Tregs. Thus, tolerance can persist much longer than the Tregs used to induce tolerance. These preclinical data provide strong rationale to harness these potent properties of Tregs for the induction of transplantation tolerance.

Treg numbers can be increased through direct infusion of Tregs or by promotion of the expansion of endogenous Tregs; each strategy has its merits and challenges. Transplantation provides an ideal scenario for direct Treg infusion when the identity of target antigens and the time of antigen exposure are known. Compared with promoting endogenous Tregs, Treg cell therapy offers the advantages of controllable Treg specificity, dose, and therapy timing. The recent advance in large-scale manufacturing of human polyclonal and donor alloantigen-reactive Tregs has made it feasible to evaluate Treg therapy for transplantation in humans (25-29). It should be mentioned that experiences with Treg cell therapy in large animal models have been very limited. The few publications of Treg cell infusion in nonhuman primates report conflicting findings regarding cell persistence after infusion and the ability of the infused cells to induce transplant tolerance (30-34). Nonetheless, it is evident from these reports that the immunological makeup of larger animals and humans is more complex than that of laboratory rodents, and efforts in translating Treg therapy to the clinic should take these potential differences into consideration.

\section{Clinical trials of Tregs in transplantation}

General considerations. In solid organ transplantation, clinical outcomes of liver and kidney transplant with the current standard of care are superior to those of pancreas, lung, and small intestine transplants (35). Although these other transplants are in need of improvements, kidney and liver transplantation have the highest volume and most clinical experience for testing novel therapies. As of January 2017, one transplant Treg therapy trial had been published (36) and 12 active trials were registered on ClinicalTrials.gov (Table 1 ) in liver and kidney transplantation $(37,38)$.

Three important clinical decisions in designing trials for Treg therapy are whom to treat, when to treat, and how to treat. An ideal design for early Treg therapy clinical trials in solid organ transplantation would allow assessment of safety as well as signals of efficacy. Initial clinical trials need to provide a number of clarifications on the best use of Tregs. First, what is the optimum dosage of Tregs? Second, are alloantigen-reactive Tregs required for efficacy as compared with polyclonal Tregs, which are easier to manufacture? Third, can a single Treg infusion induce infectious tolerance, thereby extending the biological effects of Tregs indefinitely, or is there a need for frequent dosing to replenish Tregs? Fourth, what are the specific requirements, particularly for adjunct immunosuppressants, to maximize the survival and function of infused Tregs?

Trial designs for kidney versus liver transplants. Kidney and liver transplantations have distinct immunological features that should be considered in trial design. Tolerance can be more easily achieved in liver transplantation than in kidney transplantation. Risk of liver graft damage, should rejection occur, can be limited because of the ability of the liver to regenerate. In contrast, damage to kidney grafts accumulates with each rejection episode and can lead to reduced graft function and eventual graft loss. Clinical trials of immunosuppression withdrawal in liver transplantation have found that rates of spontaneous graft tolerance increase with time after transplant, ranging from $10 \%$ two to six years after transplantation to more than $50 \%$ ten years or more after transplantation (2). Thus, liver transplantation provides a better opportunity for more ambitious protocols that incorporate complete drug withdrawal. In contrast, kidney transplant recipients have an infinitesimally low rate of spontaneous tolerance, and drug withdrawal or even modest strategies of CNI withdrawal or elimination of steroids in patients with low rejection risk are associated 

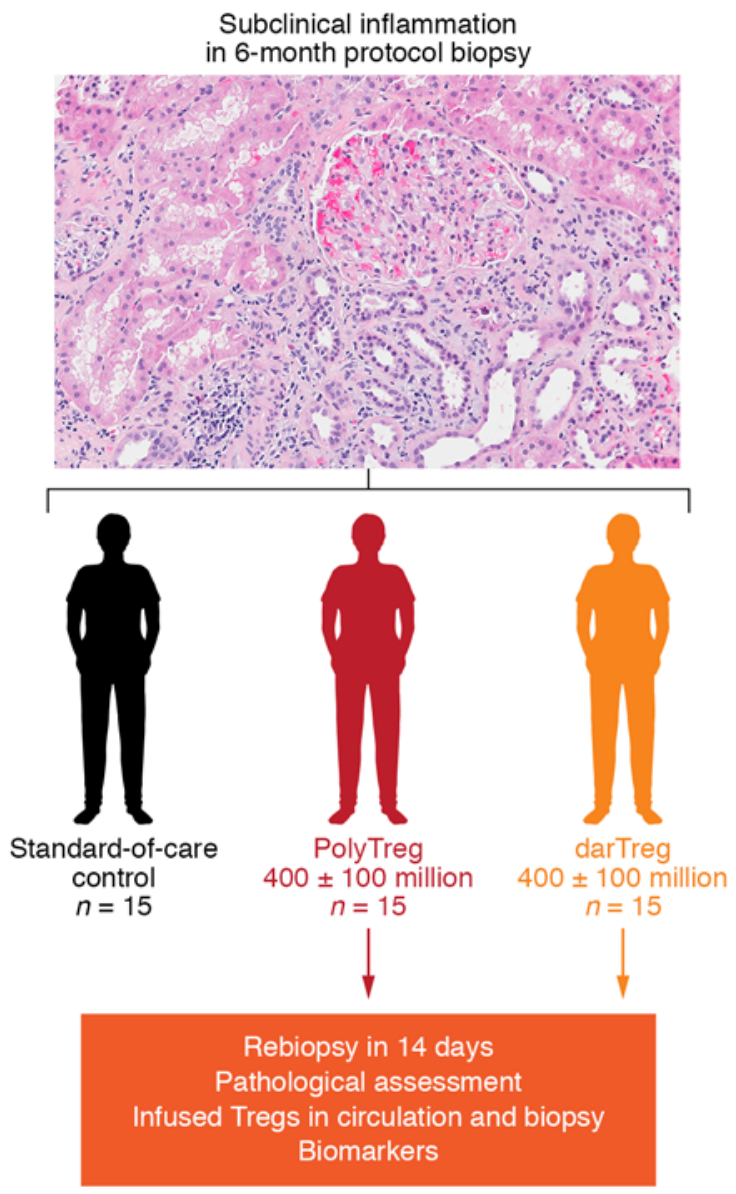

Figure 2. TASK study design. PolyTreg, polyclonal Treg; darTreg, donor alloantigen-reactive Treg.

with rejection and de novo formation of donor-specific antibodies (39-41). Even the use of the costimulation inhibitor belatacept in a $\mathrm{CNI}$-free regimen is associated with greater incidence and severity of acute rejection, although belatacept provides long-term outcome benefits (42). Thus, there are currently insufficient data to justify complete immunosuppression withdrawal in conjunction with Treg infusions in kidney transplant recipients. Moreover, the excellent early outcome results and low rejection rate of kidney transplants make it nearly impossible to test the ability of Tregs to prevent rejection when delivered at the time of transplantation (43). These issues make it more challenging to design informative Treg therapy trials in kidney transplantation.

Selection of concomitant immunosuppression. Another important challenge in designing Treg protocols is the selection of concomitant immunosuppression. There is a wide array of immunosuppressive drugs for preventing transplant rejection. Differential impacts of the various immunosuppressive drugs on Tregs versus graft-rejecting Teffs have been comprehensively reviewed (8). Most immunosuppressive drugs interfere with Treg function or survival, and the negative effects are more pronounced at higher drug doses. For example, CNIs inhibit the nuclear translocation of nuclear factor of activated T cells (NFAT), which is essential for expression of IL-2 by activated T cells (44). NFAT is also an important transcription factor for Treg identity and function (45). There is evidence that Treg induction and function require less NFAT, making Tregs relatively resistant to CNIs (46); however, persistent high doses of CNIs may impair Tregs by inhibiting Tconv production of IL-2, an essential Treg survival and stability factor (47). mTOR inhibitors have been widely regarded as "Treg-friendly" drugs. Although the mTOR inhibitor sirolimus negatively impacts Treg expansion in vitro, Tregs are relatively more resistant to sirolimus than Tconvs. Thus, inclusion of sirolimus during the manufacture of clinical-grade Tregs improves the purity of the products (8). Use of mTOR inhibitors in vivo may also favor Tregs, and deficiency in the PI3K/mTOR signaling pathway forces Tconvs to adopt a Treg phenotype after activation, while overactive PI3K pathway signaling destabilizes Tregs $(48,49)$. However, completely abolishing mTOR signaling, particularly by the sirolimus- and everolimus-targeted TORC1 pathway, impairs Treg function (50), suggesting that the pro-Treg effect of mTOR inhibitors is dosedependent. Currently, there are no drugs that selectively target Tconvs or Tregs. Experimental data suggest that combinations of low doses of these drugs are the best option to preferentially preserve Tregs while inhibiting Tconvs.

Timing of Treg infusion. Most preclinical models of Treg therapy infuse Tregs near the time of transplantation. The rationale for this design is that early infusion of Tregs allows the cells to prevent Teff activation. Ischemia-induced inflammation in the organs may attract infused Tregs, but several factors may impede Treg function or stability. Induction therapy with depleting agents given at the time of transplant will deplete the infused Tregs. CNIs are usually maintained at high levels early after transplant to prevent early acute rejection and will likely decrease the survival and/ or fitness of Tregs. Moreover, intense inflammation shortly after transplantation overrides Treg suppression and prevents de novo Treg induction and can convert Tregs into proinflammatory effectors $(51,52)$. Thus, Tregs are most vulnerable when introduced at the time of transplantation. Ideally, Treg therapy should be used when the grafts are relatively quiescent or inflammation is at subclinical levels. These settings may also allow the use of an immunosuppression regimen that either minimizes CNI dose or is more supportive of Treg function and homeostasis.

Treg specificity. Treg specificity and method of manufacture may impact the safety and efficacy of the cells. In the absence of ex vivo expansion, purified Tregs are simple to manufacture, but the number of cells collected from patients is limited (53). To effectively change the balance between Tconvs and Tregs, a Tconv depletion step is needed between Treg collection and infusion. Ex vivo expansion elevates Treg numbers and offers an opportunity to select for donor-reactive Tregs. Good manufacturing practicecompliant (GMP-compliant) processes have been developed for polyclonally expanded and donor alloantigen-expanded Tregs (26-29). Mouse studies have demonstrated that both types of Tregs can prevent transplant rejection, but more polyclonally expanded than donor alloantigen-expanded Tregs are needed (24, 54-56). Donor alloantigen-reactive Tregs have the advantage of selectively regulating donor-specific responses. Donor alloantigenreactive Tregs can be further divided into direct and indirect Tregs based on their reactivity to intact donor MHC (direct) or donor MHC peptides presented by host APC (indirect). As with Tconvs, direct Tregs are present at higher frequency than indirect Tregs 
in a natural Treg repertoire. Data from experimental transplant models show that tolerance-promoting protocols induce indirect Treg expansion (57-59); however, direct Tregs can induce longterm graft survival without maintenance immunosuppression (24, $55)$. Ongoing and planned trials use either polyclonally expanded Tregs or direct donor alloantigen-reactive Tregs. The relative efficacy and longevity of the effects of these different types of Tregs in humans remain to be tested.

\section{Clinical trials of Treg therapy in liver transplantation}

Recently, a Treg therapy trial to induce tolerance in de novo adult living-donor liver transplant recipients has been reported (36). Patients enrolled in this trial underwent left lobe liver transplantation along with splenectomy and conventional immunosuppression. CNI, mycophenolate mofetil (MMF), and steroid were initiated at the time of transplant, along with a single dose of cyclophosphamide on day 5 after transplant. On day 13 after transplant, recipients received a single infusion of Treg-enriched autologous peripheral blood mononuclear cells (PBMCs) that had been stimulated with irradiated donor PBMCs in the presence of anti-CD80 and anti-CD86 antibodies for 13 days. MMF and steroid were stopped 1 month after transplant, and CNI was withdrawn gradually over a 1-year period starting at 6 months after transplant. Seven of ten treated patients achieved complete cessation of immunosuppression without rejection for 16-33 months. By comparison, spontaneous liver transplant tolerance is $15 \%$ in the first 2 years after transplant (60).

The ongoing Donor-Alloantigen-Reactive Regulatory T Cell in Liver Transplantation (deLTa) trial (NCTO2188719, ClinicalTrials.gov) design was based on a preclinical concept showing that depletion of $\mathrm{CD}^{+}$mononuclear cells combined with donor alloantigen-reactive Treg infusion can induce transplant tolerance (20). The protocol includes the use of anti-thymocyte globulin as a depletion agent given shortly after transplant. The patients will be converted to a reduced CNI regimen with the addition of an mTOR inhibitor before infusion of autologous donor alloantigen-reactive Tregs approximately 3 months after Treg infusion. The trial will test three doses of Tregs at 50, 200, and 800 million cells per total dose. Tregs will be labeled with deuterium to permit tracking of the infused cells (PMD: 26606968).

Similar trials of Treg therapy in liver transplantation include the Safety and Efficacy Study of Regulatory T cell Therapy in Liver Transplant Patients (ThRIL trial; NCT02166177), which uses polyclonally expanded Tregs and gradual immunosuppression withdrawal after Treg infusion. An ongoing trial in Nanjing, China (NCT01624077), also uses polyclonally expanded Tregs given early after transplantation followed by immunosuppression withdrawal. The ARTEMIS trial (NCTO2474199) has a different design in that patients with stable liver graft function 2-6 years after transplant are selected for participation. Approximately 400 million donor alloantigen-reactive Tregs will be given during the course of immunosuppression withdrawal. The aim of the trial is to determine whether donor alloantigen-reactive Tregs will allow more than $15 \%$ of patients to discontinue immunosuppression. All of these trials are ongoing, and results will be greatly anticipated in the next several years.

\section{Clinical trials of Treg therapy in kidney transplantation}

In contrast to liver transplant patients, testing the efficacy of Tregs in kidney transplant recipients is both more challenging and riskier. Currently there are five ongoing trials with Treg therapies, four in the ONE Study consortium (Table 1). Two other trials have been completed and presented in abstract form: Trial of Adoptive Immunotherapy with TRACT to Prevent Rejection in Living Donor Kidney Transplant Recipients (TRACT; NCT02145325) and the Pilot Trial of Polyclonal Treg Adoptive Therapy for Control of Subclinical Transplant Inflammation (TASKp; NCT02088931).

The TRACT trial. The TRACT trial is a phase I study in livingdonor kidney transplant recipients (38). The trial was designed as a nonrandomized dose-ranging study with three tiers of cell dosing. The Treg product was produced from the Tregs obtained from leukapheresis before transplantation and expanded over 3 weeks using CD3/CD28 beads, IL-2, and sirolimus. The release criteria required greater than $70 \% \mathrm{CD} 4^{+} \mathrm{CD} 25^{+}$cells, fewer than $10 \% \mathrm{CD}^{+}$and $\mathrm{CD} 19^{+}$cells, and greater than $50 \%$ suppression of Teff proliferation in vitro. Kidney transplant recipients received alemtuzumab induction to achieve lymphodepletion and tacrolimus/MMF-based immunosuppression. Subjects were converted from tacrolimus to sirolimus at 30 days after kidney transplant to provide a milieu conducive to the survival of infused Tregs. Tregs were infused 60 days after transplantation. Nine subjects have been enrolled, and all have received TRACT. There have been no serious adverse events attributable to TRACT in any subject. Protocol biopsies performed after TRACT have not shown rejection. There have been no infectious complications. Immunophenotypic analysis of the subjects shows a significant (9- to 20-fold) increase in the percentage of circulating $\mathrm{CD} 4^{+} \mathrm{CD} 127^{-} \mathrm{CD} 25^{\mathrm{hi}} \mathrm{Foxp} 3^{+}$cells in peripheral blood after TRACT. The authors concluded that the safety of the phase I trial allows planning for a phase II trial; however, because the Tregs were infused following severe T cell depletion, the percentage increase in Tregs may not be meaningful or clinically relevant if the absolute number of Tregs is very low.

The TASKp trial. The TASKp trial is a pilot trial to determine the feasibility of expanding polyclonal Tregs ex vivo after isolation from transplant recipients on immunosuppression in the presence of subclinical graft inflammation on the protocol biopsy at 6 months following transplantation (37). Each of the three patients received a single dose of 320 million polyclonal Tregs. The study showed that Treg infusions were safe and were not associated with acute side effects, nor did infusion precipitate episodes of acute rejection. Kidney biopsies performed 2 weeks after the infusion showed a marked decrease in inflammation in two of three patients that was associated with a concomitant decrease in inflammatory gene expression. The deuterium label used to track the infused Tregs was found exclusively in the Treg population and not in Tconvs after infusion. The results of this pilot study suggest that maintenance immunosuppression did not negatively impact Treg pharmacokinetics or lineage stability. The data on the control of inflammation require confirmation in a larger trial.

The ONE Study. The ONE Study is a consortium of six clinical trials that are evaluating the safety of regulatory cell therapy in kidney transplantation. All six trials follow a similar clinical protocol of living-donor kidney transplant in six different clinical centers in 
Europe and the US. This consortium includes four different preparations of Tregs along with regulatory macrophages and tolerogenic dendritic cells (Table 1). The UK group of King's College London and Oxford University (NCTO2129881) uses polyclonal Tregs expanded with anti-CD3/anti-CD28 bead stimulation of magnetic-activated cell sorting-purified (MACS-purified) $\mathrm{CD} 4^{+} \mathrm{CD} 25^{+}$ Tregs in the presence of sirolimus over a 35-day period. The cells are then cryopreserved and are quality-tested before infusion. The group at Charité-Universitätsmedizin Berlin (NCTO2371434) is also evaluating polyclonal Tregs expanded ex vivo using antiCD3/anti-CD28 beads, but the cells are infused fresh, without cryopreservation. In contrast, the two US sites, the University of California, San Francisco (UCSF; NCT02244801), and Massachusetts General Hospital (MGH; NCTO2091232), are evaluating donor alloantigen-reactive Tregs. The UCSF process uses primary donor B cells to stimulate FACS-purified $\mathrm{CD} 4^{+} \mathrm{CD} 127^{\mathrm{lo} /}$ $\mathrm{CD} 25^{+}$Tregs to selectively expand the pool of donor alloantigenreactive Tregs, and secondary polyclonal stimulation with antiCD3/anti-CD28 beads to increase cell numbers (28). Donor B cells are first activated and expanded using CD40L stimulation to increase their antigenicity and ability to drive Treg expansion. The $\mathrm{MGH}$ process uses donor PBMCs as APCs to stimulate recipient PBMCs in the presence of belatacept to block costimulation. After 3 days, $\mathrm{CD} 4{ }^{+} \mathrm{CD} 25^{\text {hi }}$ Tregs are MACS-purified and used for infusion. At all the participating sites, Tregs are infused within the first 10 days after transplant and all patients receive the same immunosuppression regimen of tacrolimus, MMF, and a 3-month steroid tapering period without any induction therapy. The consortium has treated more than 35 patients in total, and all sites have completed enrollment or are still actively enrolling.

The TASK trial. Renal allograft inflammation below the threshold of rejection is a unique setting for testing the therapeutic effect of Tregs (61). Routine protocol kidney biopsies in patients with stable renal function show that $10 \%-20 \%$ have inflammatory mononuclear cell infiltration in the renal graft (62). Inflammation in protocol biopsies has been considered benign and not requiring therapy; however, recent studies have associated inflammation with subsequent deterioration of renal function and graft loss (63, 64). There is no consensus about what remedial therapy, if any, to use for subclinical inflammation. A recent randomized prospective study showed no benefit from a steroid pulse treatment (65).

The subclinical inflamed renal allograft offers several advantages for testing both the safety and the efficacy of Tregs. First, there is no current standard of care for this condition, and therefore novel therapies can be ethically tested. Second, Tregs are naturally attracted to areas of inflammation in order to reestablish immune homeostasis, and their trafficking in the allograft can be measured. Third, an efficacy signal can be obtained with shortterm follow-up rather than with the traditional outcome trials. A kidney biopsy performed after Treg infusion can be used to assess changes in inflammation, alterations in cellular infiltrate, and changes in inflammatory gene expression in the blood, kidney, and urine, which can provide additional evidence of an immune response undergoing homeostatic control. Additionally, if deuterium-labeled Tregs have migrated to the allograft, they may be detected and thereby convincingly establish their role in controlling an adverse cellular response.
The TASK trial was designed as both a safety and efficacy trial (Figure 2). The purpose of this trial is to test three hypotheses: (a) Polyclonal Treg and donor alloantigen-reactive Treg infusions are safe. The infused Tregs will increase the number of Tregs in circulation, will not convert to Tconvs, and are not associated with rejection. (b) Donor alloantigen-reactive Tregs will accumulate more effectively in the graft than polyclonal Tregs. (c) Treg infusions will suppress graft inflammation in the graft, as well as molecular markers of inflammation. To test these hypotheses, 45 recipients of living-donor kidneys who exhibited graft inflammation based on protocol biopsy at 6 months after transplant will be randomized to one of the three arms (Figure 2). The trial protocol is to infuse 400 million \pm 100 million Tregs into each patient. To some degree, the number of Tregs is arbitrary, but it was selected because it can be easily achieved with the current manufacturing protocol in patients who are on chronic immunosuppression. A very preliminary efficacy signal was observed in a preceding TASKp pilot trial (64).

\section{Perils of Treg therapy in transplantation}

While preclinical data provide a strong rationale for the use of Treg therapy to promote transplantation tolerance, clinical translation is expected to be challenging. Cell-based therapy is a completely new class of therapeutics, thus with many unknown factors. First, current trials use autologous Tregs that are specially produced for each patient; thus, manufacturing performance can be highly variable and may be influenced by patient demographics, disease status, and medications. These challenges are compounded by the shortage of GMP-grade reagents specifically designed to support Treg manufacturing. Second, cells are live drugs that can adapt to the in vivo environment after infusion. The adaptability and versatility of Tregs underlie their potency compared with conventional small molecules and biologics, but also raise safety concerns should they lose Treg identity and adopt a pathogenic Teff phenotype. Tregs are prone to destabilize in strongly inflammatory environments deprived of IL-2 $(47,51,52)$. Thus, Treg therapy designs should consider strategies to minimize risks of Treg plasticity, including increasing the stringency of product release criteria, creating a favorable in vivo environment by selecting therapy timing and concomitant immunosuppression, and closely monitoring Treg products after infusion. Third, animal models largely focus on Treg therapy efficacy in transplantation, and toxicity of the therapy is rarely investigated. None of the published Treg therapies in humans has seen infusion reactions, and the long-term impact of immunity against infections and malignancies is yet to be determined. Moreover, destabilized donor alloantigen-reactive Tregs can potentially contribute to graft rejection. These risks to patients need to be closely monitored in early trials. Lastly, the longevity of Treg-induced tolerance (if achievable) remains to be determined. Rodent studies suggest that Treg-induced tolerance can be perpetuated via infectious tolerance; thus, one infusion is sufficient for lifelong protection (66). In addition to much longer life expectancy, human patients also have much more complex immunological experiences when compared with rodents kept in a pathogen-free laboratory environment. Both time and immunological challenges may erode tolerance (67). Taken together, the enthusiasm for promoting transplant tolerance using Treg therapy 
must be balanced with the recognition of the known and unknown uncertainties in this nascent field so that a realistic development path can be envisioned.

\section{Future applications of Treg therapies}

The time has come for Treg therapies to be fully tested in organ transplantation to determine their proper role in controlling adverse alloimmune responses and to determine whether they can prolong long-term drug-free graft survival. The current clinical trials in liver and kidney transplantation will provide guidance as to what role Treg therapy can play in solid organ transplantation. Is Treg therapy safe in transplant patients? Do Tregs persist after infusion, and do they maintain their lineage identity in transplant patients receiving immunosuppression? Can Treg therapy modulate the immune system and exert long-term biological effects that allow drug minimization, drug withdrawal, or the establishment of true tolerance? Is there a need to combine Treg infusions with novel drugs or biologics that enhance or propagate Treg effects? Is there a need for more robust Treg delivery or modification of Tregs with chimeric antigen receptors (68-70)? It is clear that the current trials are the beginning of a new era in adoptive cell therapy. Thoughtful clinical trial designs combined with indepth mechanistic studies of trial samples will be instrumental in expanding our knowledge of human Treg biology and in allowing faster iterations of clinical testing to maximize the chance of therapeutic success.

\section{Acknowledgments}

This work is supported by NIH grants U01AI110658, U01AI104347, and U01AI113362. The authors thank Edward K. Geissler for providing information on ONE Study design and status update.

Address correspondence to: Flavio Vincenti, Department of Surgery, University of California San Francisco, 505 Parnassus Avenue, M884, San Francisco, California 94143, USA. Phone: 415.353.1322; Email: Flavio.Vincenti@ucsf.edu.
1. Sachs DH, Kawai T, Sykes M. Induction of tolerance through mixed chimerism. Cold Spring Harb Perspect Med. 2014;4(1):a015529.

2. Feng S. Spontaneous and induced tolerance for liver transplant recipients. Curr Opin Organ Transplant. 2016;21(1):53-58.

3. Spitzer TR, et al. Long-term follow-up of recipients of combined human leukocyte antigenmatched bone marrow and kidney transplantation for multiple myeloma with end-stage renal disease. Transplantation. 2011;91(6):672-676.

4. Tang Q, Bluestone JA. Regulatory T-cell therapy in transplantation: moving to the clinic. Cold Spring Harb Perspect Med. 2013;3(11):a015552.

5. Braza F, Durand M, Degauque N, Brouard S. Regulatory T cells in kidney transplantation: new directions? Am J Transplant. 2015;15(9):2288-2300.

6. Webber AB, Vincenti F. An update on calcineurin inhibitor-free regimens: the need persists, but the landscape has changed. Transplantation. 2016;100(4):836-843.

7. Camirand G, Riella LV. Treg-centric ciew of immunosuppressive drugs in transplantation: a balancing act. Am J Transplant. 2017;17(3):601-610.

8. Furukawa A, Wisel SA, Tang Q. Impact of immune-modulatory drugs on regulatory $\mathrm{T}$ cell. Transplantation. 2016;100(11):2288-2300.

9. Chandran S, Feng S. Current status of tolerance in kidney transplantation. Curr Opin Nephrol Hypertens. 2016;25(6):591-601.

10. Yamaguchi T, Wing JB, Sakaguchi S. Two modes of immune suppression by Foxp $3^{+}$regulatory $\mathrm{T}$ cells under inflammatory or non-inflammatory conditions. Semin Immunol. 2011;23(6):424-430.

11. Tang Q, Bluestone J. The Foxp $3^{+}$regulatory T cell: a jack of all trades, master of regulation. Nat Immunol. 2008;9(3):239-244.

12. Busse D, et al. Competing feedback loops shape IL-2 signaling between helper and regulatory $\mathrm{T}$ lymphocytes in cellular microenvironments. Proc Natl Acad Sci U S A. 2010;107(7):3058-3063.

13. Walker LS, Sansom DM. The emerging role of CTLA4 as a cell-extrinsic regulator of T cell responses. Nat Rev Immunol. 2011;11(12):852-863.
14. Cao X, et al. Granzyme B and perforin are important for regulatory $\mathrm{T}$ cell-mediated suppression of tumor clearance. Immunity. 2007;27(4):635-646.

15. Campbell DJ, Koch MA. Treg cells: patrolling a dangerous neighborhood. Nat Med. 2011;17(8):929-930.

16. Game DS, Lechler RI. Pathways of allorecognition: implications for transplantation tolerance. Transpl Immunol. 2002;10(2-3):101-108.

17. Veerapathran A, Pidala J, Beato F, Yu XZ, Anasetti C. Ex vivo expansion of human Tregs specific for alloantigens presented directly or indirectly. Blood. 2011;118(20):5671-5680.

18. Zhang N, et al. Regulatory T cells sequentially migrate from inflamed tissues to draining lymph nodes to suppress the alloimmune response. Immunity. 2009;30(3):458-469.

19. Jiang S, Herrera O, Lechler RI. New spectrum of allorecognition pathways: implications for graft rejection and transplantation tolerance. Curr Opin Immunol. 2004;16(5):550-557.

20. Lechler RI, Garden OA, Turka LA. The complementary roles of deletion and regulation in transplantation tolerance. Nat Rev Immunol. 2003;3(2):147-158.

21. Wood KJ, Sakaguchi S. Regulatory T cells in transplantation tolerance. Nat Rev Immunol. 2003;3(3):199-210.

22. Fan Z, et al. In vivo tracking of 'color-coded' effector, natural and induced regulatory $\mathrm{T}$ cells in the allograft response. Nat Med. 2010;16(6):718-722.

23. Raimondi G, et al. Mammalian target of rapamycin inhibition and alloantigen-specific regulatory T cells synergize to promote long-term graft survival in immunocompetent recipients. J Immunol. 2010;184(2):624-636.

24. Lee K, Nguyen V, Lee KM, Kang SM, Tang Q. Attenuation of donor-reactive T cells allows effective control of allograft rejection using regulatory $\mathrm{T}$ cell therapy. Am J Transplant. 2014;14(1):27-38.

25. Riley JL, June CH, Blazar BR. Human T regulatory cell therapy: take a billion or so and call me in the morning. Immunity. 2009;30(5):656-665.
26. Putnam AL, et al. Expansion of human regulatory T-cells from patients with type 1 diabetes. Diabetes. 2009;58(3):652-662.

27. Hippen $\mathrm{KL}$, et al. Massive ex vivo expansion of human natural regulatory $\mathrm{T}$ cells (T(regs)) with minimal loss of in vivo functional activity. Sci Transl Med. 2011;3(83):83ra41.

28. Putnam AL, et al. Clinical grade manufacturing of human alloantigen-reactive regulatory $\mathrm{T}$ cells for use in transplantation. Am J Transplant. 2013;13(11):3010-3020.

29. Safinia N, et al. Successful expansion of functional and stable regulatory $\mathrm{T}$ cells for immunotherapy in liver transplantation. Oncotarget. 2016;7(7):7563-7577.

30. Bashuda H, et al. Renal allograft rejection is prevented by adoptive transfer of anergic T cells in nonhuman primates. J Clin Invest. 2005;115(7):1896-1902.

31. Ma A, et al. Adoptive transfer of $\mathrm{CDR}^{+} \mathrm{CD} 25^{+}$reg ulatory cells combined with low-dose sirolimus and anti-thymocyte globulin delays acute rejection of renal allografts in Cynomolgus monkeys. Int Immunopharmacol. 2011;11(5):618-629.

32. Singh K, et al. Superiority of rapamycin over tacrolimus in preserving nonhuman primate Treg half-life and phenotype after adoptive transfer. Am J Transplant. 2014;14(12):2691-2703.

33. Zhang $\mathrm{H}$, et al. Sequential monitoring and stability of ex vivo-expanded autologous and nonautologous regulatory $\mathrm{T}$ cells following infusion in nonhuman primates. Am J Transplant. 2015;15(5):1253-1266.

34. Ezzelarab MB, et al. Regulatory T cell infusion can enhance memory t cell and alloantibody responses in lymphodepleted nonhuman primate heart allograft recipients. Am J Transplant. 2016;16(7):1999-2015.

35. [No authors listed]. OPTN/SRTR 2015 Annua Data Report: Introduction. Am J Transplant. 2017;17(suppl 1):11-20.

36. Todo S, et al. A pilot study of operational tolerance with a regulatory T-cell-based cell therapy in living donor liver transplantation. Hepatology. 
2016;64(2):632-643.

37. Chandran S, et al. Infusion of Polyclonal Tregs Modulates Subclinical Kidney Transplant Inflammation: Final Report of TASK Pilot Trial. [abstract]. Am J Transplant. 2017;17(suppl 3). http://atcmeetingabstracts.com/abstract/infusion-of-polyclonal-tregs-modulates-subclinicalkidney-transplant-inflammation-final-report-oftask-pilot-trial/. Accessed May 23, 2017.

38. Skaro A, et al. Results of a phase 1 trial of Treg adoptive cell transfer (TRACT) in de novo living donor kidney transplant recipients. [abstract]. Am J Transplant. 2016;16(suppl 3). http:// atcmeetingabstracts.com/abstract/results-of-aphase-1-trial-of-treg-adoptive-cell-transfer-tractin-de-novo-living-donor-kidney-transplantrecipients/. Accessed May 23, 2017.

39. Massart A, et al. The DESCARTES-Nantes survey of kidney transplant recipients displaying clinical operational tolerance identifies 35 new tolerant patients and 34 almost tolerant patients. Nephrol Dial Transplant. 2016;31(6):1002-1013.

40. Hricik DE, et al. Adverse outcomes of tacrolimus withdrawal in immune-quiescent kidney transplant recipients. JAm Soc Nephrol. 2015;26(12):3114-3122.

41. Vincenti F, et al. A randomized, multicenter study of steroid avoidance, early steroid withdrawal or standard steroid therapy in kidney transplant recipients. Am J Transplant. 2008;8(2):307-316.

42. Vincenti F. Belatacept and long-term outcomes in kidney transplantation. N Engl JMed. 2016;374(26):2600-2601.

43. Matas AJ, et al. OPTN/SRTR 2013 Annual Data Report Kidney. Am J Transplant. 2015;15(suppl 2):1-34.

44. Macian F. NFAT proteins: key regulators of T-cell development and function. Nat Rev Immunol. 2005;5(6):472-484.

45. Oh-hora M, Rao A. The calcium/NFAT pathway: role in development and function of regulatory $\mathrm{T}$ cells. Microbes Infect. 2009;11(5):612-619.

46. Vaeth M, et al. Dependence on nuclear factor of activated T-cells (NFAT) levels discriminates conventional T cells from Foxp3+ regulatory T cells. Proc Natl Acad Sci US A. 2012;109(40):16258-16263.
47. Malek TR, Castro I. Interleukin-2 receptor signaling: at the interface between tolerance and immunity. Immunity. 2010;33(2):153-165.

48. Haxhinasto S, Mathis D, Benoist C. The AKTmTOR axis regulates de novo differentiation of CD4 ${ }^{+}$Foxp3 $^{+}$cells. JExp Med. 2008;205(3):565-574.

49. Huynh A, et al. Control of PI(3) kinase in Treg cells maintains homeostasis and lineage stability. Nat Immunol. 2015;16(2):188-196.

50. Zeng H, Yang K, Cloer C, Neale G, Vogel P, Chi H. mTORC1 couples immune signals and metabolic programming to establish $\mathrm{T}$ (reg)-cell function. Nature. 2013;499(7459):485-490.

51. Hanidziar D, Koulmanda M. Inflammation and the balance of Treg and Th17 cells in transplant rejection and tolerance. Curr Opin Organ Transplant. 2010;15(4):411-415.

52. Kim JI, et al. Regulatory T-cell counter-regulation by innate immunity is a barrier to transplantation tolerance. Am J Transplant. 2009;9(12):2736-2744.

53. Tang Q, Lee K. Regulatory T-cell therapy for transplan tation: how many cells do we need? Curr Opin Organ Transplant. 2012;17(4):349-354.

54. Tsang JY, et al. Conferring indirect allospecificity on $\mathrm{CD} 4^{+} \mathrm{CD} 25^{+}$Tregs by TCR gene transfer favors transplantation tolerance in mice. J Clin Invest. 2008;118(11):3619-3628.

55. Joffre $\mathrm{O}$, et al. Prevention of acute and chronic allograft rejection with $\mathrm{CD} 4^{+} \mathrm{CD} 25^{+} \mathrm{Foxp}^{+}$regulatory T lymphocytes. Nat Med. 2008;14(1):88-92.

56. Sagoo P, Ali N, Garg G, Nestle FO, Lechler RI, Lombardi G. Human regulatory T cells with alloantigen specificity are more potent inhibitors of alloimmune skin graft damage than polyclonal regulatory T cells. Sci Transl Med. 2011;3(83):83ra42.

57. Wise MP, Bemelman F, Cobbold SP, Waldmann $\mathrm{H}$. Linked suppression of skin graft rejection can operate through indirect recognition. JImmunol. 1998;161(11):5813-5816.

58. Ochando JC, et al. Alloantigen-presenting plasmacytoid dendritic cells mediate tolerance to vascularized grafts. Nat Immunol. 2006;7(6):652-662.

59. Verginis P, McLaughlin KA, Wucherpfennig KW, von Boehmer H, Apostolou I. Induction of antigen-specific regulatory $\mathrm{T}$ cells in wild-type mice: visualization and targets of suppression. Proc Natl Acad Sci U S A. 2008;105(9):3479-3484.

60. Porrett $P$, Shaked A. The failure of immunosuppression withdrawal: patient benefit is not detectable, inducible, or reproducible. Liver Transpl. 2011;17(suppl 3):S66-S68.

61. Solez K, et al. Banff 07 classification of renal allograft pathology: updates and future directions. Am J Transplant. 2008;8(4):753-760.

62. Gloor JM, et al. Subclinical rejection in tacrolimustreated renal transplant recipients. Transplantation. 2002;73(12):1965-1968.

63. Moreso F, et al. Subclinical rejection associated with chronic allograft nephropathy in protocol biopsies as a risk factor for late graft loss. Am J Transplant. 2006;6(4):747-752.

64. Cosio FG, Grande JP, Wadei H, Larson TS, Griffin MD, Stegall MD. Predicting subsequent decline in kidney allograft function from early surveillance biopsies. Am J Transplant. 2005;5(10):2464-2472.

65. Flores-Mendoza A, et al. The treatment of borderline lesion in protocol biopsies did not improve the outcome in kidney transplant recipients. Am J Transplant. 2016;16(suppl 3). http://atcmeetingabstracts.com/abstract/thetreatment-of-borderline-lesion-in-protocol-biopsies-did-not-improve-the-outcome-in-kidneytransplant-recipients/. Accessed June 2, 2017.

66. Kendal A, et al. Sustained suppression by Foxp3+ regulatory $\mathrm{T}$ cells is vital for infectious transplantation tolerance. JExp Med. 2011;208(10):2043-2053.

67. Young JS, et al. Erosion of transplantation tolerance after infection. Am J Transplant. 2017;17(1):81-90.

68. MacDonald KG, et al. Alloantigen-specific regulatory $\mathrm{T}$ cells generated with a chimeric antigen receptor. J Clin Invest. 2016;126(4):1413-1424.

69. Boardman DA, et al. Expression of a chimeric antigen receptor specific for donor HLA class I enhances the potency of human regulatory $\mathrm{T}$ cells in preventing human skin transplant rejection. Am J Transplant. 2017;17(4):931-943.

70. Noyan F, et al. Prevention of allograft rejection by use of regulatory $\mathrm{T}$ cells with an $\mathrm{MHC}$-specific chimeric antigen receptor. Am J Transplant. 2017;17(4):917-930. 